\title{
Existence of positive solutions of singular $p$-Laplacian equations in a ball
}

Fang $\mathrm{Li}^{\mathrm{a}}$, Zuodong Yang ${ }^{\mathrm{a}, \mathrm{b}, *}$

anstitute of Mathematics, School of Mathematical Sciences, Nanjing Normal University, Jiangsu Nanjing 210046, China.

${ }^{b}$ College of Zhongbei, Nanjing Normal University, Jiangsu Nanjing 210046, China.

\author{
This paper is dedicated to Professor Ljubomir Ćirić \\ Communicated by Professor V. Berinde
}

\begin{abstract}
In this paper, we investigate singular $p$-Laplacian equations of the form $\triangle_{p} u+f(x, \nabla u) u^{-\lambda}=0$ with zero Dirichlet boundary condition in a ball $B \subset \mathbf{R}^{N}$, where $p>1, \lambda>0$, and give a sufficient condition for the equation to have a positive solution, by means of a supersolution and a subsolution.(C)2012 NGA. All rights reserved.
\end{abstract}

Keywords: Positive solution; singular equation; supersolution and subsolution.

2010 MSC: Primary 58J10; Secondary 35N20.

\section{Introduction}

We shall establish the results on the existence of positive solutions of singular $p$-Laplacian equations

$$
\begin{gathered}
\triangle_{p} u+f(x, \nabla u) u^{-\lambda}=0, \quad x \in B \subset \mathbf{R}^{N}, \\
u=0, \quad x \in \partial B,
\end{gathered}
$$

where $p>1, \triangle_{p} u:=\operatorname{div}\left(|\nabla u|^{p-2} \nabla u\right), x=\left(x_{1}, x_{2}, \cdots, x_{N}\right), \nabla$ is the gradient operator, $B$ is an open ball centered at the origin of $\mathbf{R}^{N}, \partial B$ is the boundary of $B, \lambda>0$ is a constant, and $f(x, u)$ is locally Hölder continuous with exponent $\theta \in(0,1)$.

\footnotetext{
${ }^{*}$ Corresponding author

Email addresses: lifang101216@126.com (Fang Li), zdyang_jin@263.net (Zuodong Yang)
} 
Equations of the above form are mathematical models occurring in studies of the $p$-Laplace equation, generalized reaction-diffusion theory [19], non-Newtonian fluid theory [2, 25], non-Newtonian filtration [18] and the turbulent flow of a gas in porous medium [8]. In the non-Newtonian fluid theory, the quantity $\mathrm{p}$ is characteristic of the medium.Media with $p>2$ are called dilatant fluids and those with $p<2$ are called pseudoplastics. If $p=2$, they are Newtonian fluids.

During the past three decades, singular elliptic equations have been paid much attention by many mathematicians. In particular, the existence and the uniqueness of positive solutions of the following singular elliptic boundary value problems

$$
\begin{cases}-\Delta u=\eta(x) u^{-\lambda}, & x \in \Omega, \\ u(x)=0, & x \in \partial \Omega,\end{cases}
$$

where $\eta(x) \geq 0$, in $\Omega, \lambda>0$, have been studied widely, see for instance [9, 21, 17] and references therein.

In [4], the authors studied general singular elliptic equation of the following

$$
\begin{cases}-\Delta u+h(u) \frac{|\nabla u|^{2}}{u^{\gamma}}=f, & x \in \Omega, \\ u(x)=0, & x \in \partial \Omega\end{cases}
$$

where $\Omega$ is an open bounded subset of $\mathbf{R}^{N}, \gamma>0$ and $f$ is a function which is strictly positive on every compactly contained subset of $\Omega$. They prove that the condition $\gamma<2$ is necessary and sufficient for the existence of solutions in $H_{0}^{1}(\Omega)$ for every sufficiently regular $f$ as above.

Recently, Ahmed Mohammed [1] studied of the existence of the positive solution of the equation

$$
\begin{cases}-\Delta_{p} u=\lambda f(x, u), & x \in \Omega, \\ u>0, & x \in \Omega, \\ u(x)=0, & x \in \partial \Omega\end{cases}
$$

where $\Omega \subset \mathbf{R}^{N}$ is a $C^{1, \varpi}$ bounded domain, for some $0<\varpi<1, f: \Omega \times(0, \infty) \rightarrow[0, \infty)$ is a suitable function and allowed to be singular, $\lambda>0$.

Yao and Zhou [20] shows the existence of positive solutions for the one-dimensional singular $p$-Laplacian

$$
\left\{\begin{array}{l}
{\left[\Phi_{p}\left(\psi^{\prime}\right)\right]^{\prime}-\lambda \frac{\left|\psi^{\prime}\right|^{p}}{\psi}+f(t)=0, \quad 0<t<1,} \\
\psi(1)=\psi(0)=\psi^{\prime}(1)=\psi^{\prime}(0)=0
\end{array}\right.
$$

where $\Phi_{p}(s)=|s|^{p-2} s, p \geq 2, \lambda>0, f(t) \in C[0,1]$ and $f(t)>0$ on $[0,1]$.

For the other results of singular elliptic equations, see [13, 14, 16, 22, 23, 24] and the references therein.

Motivated by the results of the above cited papers, we shall attempt to treat such equation (1.1)(1.2), the results of the semilinear equations are extending the quasilinear ones. We can find the related results for $p=2$ in [10]. The main differences between $p=2$ and $p \neq 2$ are known in [5, 6]. When $p \neq 2$, the problem becomes more complicated since certain nice properties inherent to the case $p=2[3]$ seem to be lost or at least difficult to verify. The main differences between $p=2$ and $p \neq 2$ can be found in [5, 6, 26].

This work is organized as follows: In Section 2, we give Several results and lemmas. In Section 3, we give our main results and its proof. 


\section{Several Results and Lemmas}

Before we prove the main results, we need the following lemmas. For (1.1)-(1.2), the following hypotheses on $f$ are adopted.

$\left(B_{1}\right) . f: B \times \mathbf{R}^{N} \rightarrow(0, \infty)$ is locally Hölder continuous with exponent $\theta \in(0,1)$, and $f(x, p)$ is continuously differentiable in $p$. For every compact region $\Omega \subset B$, there exists an ordinary number $\rho_{\Omega}$, such that $|f(x, t)| \leq \rho_{\Omega}\left(1+|t|^{p}\right), x \in \Omega, t \in \mathbf{R}^{N}$.

Lemma 2.1. [11] Suppose that a function $f$ satisfies $\left(B_{1}\right)$, and that there exist a supersolution $v$ and subsolution $w$ of Eq. (1.1)-(1.2) such that $0<w(x)<v(x) ; x \in B$; then Eq. (1.1)-(1.2) has a solution $u$ and $w(x)<u(x)<v(x), x \in B$.

To establish the supersolution and subsolution, we firstly consider that the function $f$ is radially symmetric, that is $f=F(|x|,|\nabla u|)$. Thus, we introduce the following radial problem

$$
\begin{gathered}
\left(r^{N-1}\left|w^{\prime}\right|^{p-2} w^{\prime}\right)^{\prime}+r^{N-1} F\left(r,\left|w^{\prime}\right|\right) w^{-\lambda}=0, \quad 0<r<1, \\
w^{\prime}(0)=0, \quad w(1)=0 .
\end{gathered}
$$

We assume that the function $F$ satisfies the following hypotheses.

$\left(A_{1}\right) \cdot F:[0,1) \times[0, \infty) \rightarrow(0, \infty)$ is continuous, $F(t, z)$ is continuously differentiable in $z \geq 0$. For each fixed $t \in[0,1), F(t, z)$ is strictly increasing in $z \geq 0$;

$\left(A_{2}\right)$. There exists a positive constant $M>0$ satisfying

$$
\int_{0}^{1}\left(\int_{0}^{s}\left(\frac{t}{s}\right)^{N-1} F(t, d) d t\right)^{\frac{1}{p-1}} d s \leq M,
$$

which holds uniformly for every $d \geq 0$.

$\left(A_{3}\right)$. For all $\epsilon>0$, there exists $\delta>0$, such that $1-\delta<r<1$, the following inequality

$$
\int_{r}^{1}\left(\int_{0}^{s}\left(\frac{t}{s}\right)^{N-1} F(t, d) d t\right)^{\frac{1}{p-1}} d s \leq \epsilon
$$

holds uniformly for every $d \geq 0$.

We study the existence of positive solutions of boundary value problems(2.1)-(2.2) by the shooting method. We consider that the unique positive solution of the initial value problem

$$
\begin{gathered}
\left(r^{N-1}\left|w^{\prime}\right|^{p-2} w^{\prime}\right)^{\prime}+r^{N-1} F\left(r,\left|w^{\prime}\right|\right) w^{-\lambda}=0, \\
w(0)=\alpha, \quad w^{\prime}(0)=0,
\end{gathered}
$$

where $\alpha>0$ is a parameter. Condition $\left(A_{1}\right)$ implies that problems (2.3)-(2.4) have a unique positive solution $w_{\alpha}(r) \in C^{1}\left[0, T_{\alpha}\right) \cap C\left[0, T_{\alpha}\right], r \in\left[0, T_{\alpha}\right)$, where $\left[0, T_{\alpha}\right)$ is the maximal existence interval in $w_{\alpha}(r)$ (see [15]). Clearly, the value of $T_{\alpha}$ lies in $0<T_{\alpha} \leq 1$. If $T_{\alpha}<1$, then $w_{\alpha}(r)>0,0 \leq r<$ $T_{\alpha} ; w_{\alpha}\left(T_{\alpha}\right)=0$. Therefore, $w_{\alpha}(r)$ depends continuously on its initial value $\alpha$.

Lemma 2.2. Suppose that $F$ satisfies $\left(A_{1}\right)-\left(A_{3}\right)$, let $\alpha$ and $\beta$ be positive numbers satisfying $\alpha>\beta$. If $w_{\beta}(r)$ exists on $[0, T),(0 \leq T<1)$, then $w_{\alpha}(r)$ also exists on $[0, T)$ and satisfies

$$
\begin{aligned}
& w_{\alpha}(r)>w_{\beta}(r), \quad r \in[0, T), \\
& w_{\alpha}^{\prime}(r)>w_{\beta}^{\prime}(r), \quad r \in[0, T) .
\end{aligned}
$$


Proof. We prove that lemma 2.2 in three steps.

Step 1. Assume that $w_{\alpha}(r)$ and $w_{\beta}(r)$ are defined on $[0, T)$. Then

$$
w_{\alpha}^{\prime}(r)>w_{\beta}^{\prime}(r), \quad r \in[0, T) .
$$

By (2.3)-(2.4) and $\left(A_{1}\right)$, we obtain

$$
\left|w_{\alpha}^{\prime}(r)\right|^{p-2} w_{\alpha}^{\prime}(r)=-\int_{0}^{r}\left(\frac{s}{r}\right)^{N-1} F\left(s,\left|w_{\alpha}^{\prime}(s)\right|\right) w_{\alpha}^{-\lambda}(s) d s<0 .
$$

Therefore $w_{\alpha}(r)$ is strictly decreasing and $\left|w_{\alpha}^{\prime}(r)\right|=-w_{\alpha}^{\prime}(r)$. Hence,

$$
\begin{aligned}
& \left|w_{\alpha}^{\prime}(r)\right|^{p-1}-\left|w_{\beta}^{\prime}(r)\right|^{p-1}= \\
& \int_{0}^{r}\left(\frac{s}{r}\right)^{N-1}\left[F\left(s,\left|w_{\alpha}^{\prime}(s)\right|\right) w_{\alpha}^{-\lambda}(s)-F\left(s,\left|w_{\beta}^{\prime}(s)\right|\right) w_{\beta}^{-\lambda}(s)\right] d s, \quad r \in[0, T) .
\end{aligned}
$$

Choose a positive number $\gamma$ such that $\beta<\gamma<\alpha$. Since $w_{\alpha}(r)$ is continuous and $w_{\alpha}(0)=\alpha, r \in$ $[0, T)$, there exists $r_{0}>0\left(0<r_{0}<T\right)$ satisfying $w_{\alpha}(r)>\gamma, 0 \leq r \leq r_{0}$, and

$$
\begin{aligned}
& F\left(s,\left|w_{\alpha}^{\prime}(s)\right|\right) w_{\alpha}^{-\lambda}(s)-F\left(s,\left|w_{\beta}^{\prime}(s)\right|\right) w_{\beta}^{-\lambda}(s) \\
& <F\left(s,\left|w_{\alpha}^{\prime}(s)\right|\right) \gamma^{-\lambda}-F(s, 0) \beta^{-\lambda}, \quad 0 \leq r \leq r_{0}
\end{aligned}
$$

Let $F(0,0) \beta^{-\lambda}-F(0,0) \gamma^{-\lambda}=a>0$. Since $\left[F\left(s,\left|w_{\alpha}^{\prime}(s)\right|\right) w_{\alpha}^{-\lambda}(s)\right.$ is continuous and $w_{\alpha}^{\prime}(0)=$ $w_{\beta}^{\prime}(0)=0$, there exists $\delta \in\left[0, r_{0}\right)$ such that

$$
\begin{gathered}
F\left(s,\left|w_{\alpha}^{\prime}(r)\right|\right) \gamma^{-\lambda}<F(0,0) \gamma^{-\lambda}+\frac{a}{2}, \\
F(r, 0) \beta^{-\lambda}>F(0,0) \beta^{-\lambda}-\frac{a}{2},
\end{gathered}
$$

where $0<r<\delta$. From (2.8) and the above equalities, we get

$$
F\left(s,\left|w_{\alpha}^{\prime}(s)\right|\right) w_{\alpha}^{-\lambda}(s)-F\left(s,\left|w_{\beta}^{\prime}(s)\right|\right) w_{\beta}^{-\lambda}(s)<0, \quad 0<r<\delta \leq T .
$$

It also follows (2.7) and (2.9) that $\left|w_{\alpha}^{\prime}(r)\right|^{p-1}<\left|w_{\beta}^{\prime}(r)\right|^{p-1}, \quad 0<r<\delta \leq T$. It implies that $w_{\alpha}^{\prime}(r)>w_{\beta}^{\prime}(r), \quad 0<r<\delta \leq T$. Now we prove that $\delta=T$.

If $\delta<T$, we find that $\delta_{1}: \delta<\delta_{1}<T$, such that

$$
w_{\alpha}^{\prime}(r)>w_{\beta}^{\prime}(r), \quad 0<r<\delta_{1}, \quad w_{\alpha}^{\prime}\left(\delta_{1}\right)=w_{\beta}^{\prime}\left(\delta_{1}\right) .
$$

Note that

$$
w_{\alpha}(0)-w_{\beta}(0)=\alpha-\beta, w_{\alpha}^{\prime}(r) \leq 0, w_{\beta}^{\prime}(r) \leq 0,0<r<\delta_{1} .
$$

Thus,

$$
w_{\alpha}(r)>w_{\beta}(r), \quad\left|w_{\alpha}^{\prime}(r)\right|<\left|w_{\beta}^{\prime}(r)\right|, \quad 0<r<\delta_{1} .
$$

Hence, we have

$$
F\left(r,\left|w_{\alpha}^{\prime}(r)\right|\right) w_{\alpha}^{-\lambda}(r)<F\left(r,\left|w_{\beta}^{\prime}(r)\right|\right) w_{\beta}^{-\lambda}(r), \quad 0<r<\delta_{1}
$$

and

$$
\begin{aligned}
& 0=\left|w_{\alpha}^{\prime}\left(\delta_{1}\right)\right|^{p-1}-\left|w_{\beta}^{\prime}\left(\delta_{1}\right)\right|^{p-1}= \\
& \int_{0}^{\delta_{1}}\left(\frac{s}{\delta_{1}}\right)^{N-1}\left[F\left(s,\left|w_{\alpha}^{\prime}(s)\right|\right) w_{\alpha}^{-\lambda}(s)-F\left(s,\left|w_{\beta}^{\prime}(s)\right|\right) w_{\beta}^{-\lambda}(s)\right] d s<0 .
\end{aligned}
$$


This contradiction proves that (2.6) holds.

Step 2. If $w_{\beta}(r)$ exists on $[0, T)$, then $w_{\alpha}(r)$ also exists on $[0, T)$.

In fact, we assume that the existence interval of $w_{\alpha}(r)$ is less than $[0, T)$. Since $w_{\alpha}(r)>w_{\beta}(r)$ near the origin, the curve $w_{\alpha}(r)$ is sure to intersect the curve $w_{\beta}(r)$. Suppose that the first intersection point is $t=\tau<T$. Then we have

$$
w_{\alpha}(r)>w_{\beta}(r), \quad 0 \leq r<\tau ; \quad w_{\alpha}(\tau)=w_{\beta}(\tau) .
$$

Using (2.3),(2.4), condition $\left(A_{1}\right)$ and the conclusion of step1, we get

$$
\begin{aligned}
& w_{\alpha}(\tau)-w_{\beta}(\tau)+\beta-\alpha \\
& =-\int_{0}^{\tau}\left(\int_{0}^{s}\left(\frac{t}{s}\right)^{N-1} F\left(t,\left|w_{\alpha}^{\prime}(t)\right|\right) w_{\alpha}^{-\lambda}(t) d t\right)^{\frac{1}{p-1}} d s \\
& -\int_{0}^{\tau}\left(\int_{0}^{s}\left(\frac{t}{s}\right)^{N-1} F\left(t,\left|w_{\beta}^{\prime}(t)\right|\right) w_{\beta}^{-\lambda}(t) d t\right)^{\frac{1}{p-1}} d s \geq 0 .
\end{aligned}
$$

Thus, $w_{\alpha}(\tau)-w_{\beta}(\tau) \geq \alpha-\beta>0$. This contradiction proves that $w_{\alpha}(r)$ also exists on $[0, \mathrm{~T})$.

Step 3. We prove (2.5) holds. In fact, making use of $w_{\alpha}(0)-w_{\beta}(0)=\alpha-\beta>0$ and the above conclusions, we can prove immediately that (2.5)holds. Lemma 2.2 is proved.

Lemma 2.3. Under the assumptions $\left(A_{1}\right)-\left(A_{3}\right)$, the boundary value problems (2.1)-(2.2) have a unique positive solution $w \in C^{2}([0,1)) \cap C((0,1])$.

Proof. Define the subsets $\bar{S}, \underline{S} \subset(0, \infty)$, respectively, by

$\bar{S}=\left\{\alpha>0 \mid w_{\alpha}(r)\right.$ exists on $[0,1)$ and satisfies $\left.w_{\alpha}(1)>0\right\}$

$\underline{S}=\left\{\alpha>0 \mid w_{\alpha}(r)\right.$ vanishes before $\left.r=1\right\}$.

It follows from Lemma 2.2 that for all $\alpha \in \bar{S}$ and for all $\beta \in \underline{S}, \alpha>\beta$. Thus, $\bar{S} \cap \underline{S}=\oslash$. The following results (i)-(v) are valid.

(i). $\bar{S}$ is not empty.

Choosing arbitrarily a positive number $\alpha_{1}$ such that $\frac{\alpha_{1}}{2}>M>1$. Thus, by condition $\left(A_{2}\right), \alpha_{1}$ satisfies

$$
\begin{aligned}
& \int_{0}^{1}\left(\int_{0}^{s}\left(\frac{t}{s}\right)^{N-1} F(t, d)\left(\frac{\alpha_{1}}{2}\right)^{-\lambda} d t\right)^{\frac{1}{p-1}} d s \\
& <\int_{0}^{1}\left(\int_{0}^{s}\left(\frac{t}{s}\right)^{N-1} F(t, d) d t\right)^{\frac{1}{p-1}} d s<M<\frac{\alpha_{1}}{2},
\end{aligned}
$$

which holds uniformly for every $d \geq 0$. We claim that $w_{\alpha_{1}}(r)>\frac{\alpha_{1}}{2}$, for $r \in[0,1)$. In fact, if this is not true, then there exists $r_{1} \in(0,1)$ such that

$$
w_{\alpha_{1}}(r)>\frac{\alpha_{1}}{2}, r \in\left[0, r_{1}\right) ; \quad w_{\alpha_{1}}\left(r_{1}\right)=\frac{\alpha_{1}}{2} .
$$

Making use of (2.3), (2.4), we get

$$
w_{\alpha_{1}}\left(r_{1}\right)-\alpha_{1}+\int_{0}^{r_{1}}\left(\int_{0}^{s}\left(\frac{t}{s}\right)^{N-1} F\left(t,\left|w_{\alpha_{1}}^{\prime}(t)\right|\right) w_{\alpha_{1}}^{-\lambda}(t) d t\right)^{\frac{1}{p-1}} d s=0 .
$$

Let $d_{1}=\max _{0 \leq t \leq r_{1}}\left|w_{\alpha_{1}}^{\prime}(t)\right|$. Eqs. $(2.10),(2.11)$ and condition $\left(A_{1}\right)$ can be applied to get

$$
\begin{aligned}
& \frac{\alpha_{1}}{2}=\int_{0}^{r_{1}}\left(\int_{0}^{s}\left(\frac{t}{s}\right)^{N-1} F\left(t,\left|w_{\alpha_{1}}^{\prime}(t)\right|\right) w_{\alpha_{1}}^{-\lambda}(t) d t\right)^{\frac{1}{p-1}} d s \\
& \leq \int_{0}^{r_{1}}\left(\int_{0}^{s}\left(\frac{t}{s}\right)^{N-1} F\left(t, d_{1}\right)\left(\frac{\alpha_{1}}{2}\right)^{-\lambda} d t\right)^{\frac{1}{p-1}} d s \\
& \leq \int_{0}^{1}\left(\int_{0}^{s}\left(\frac{t}{s}\right)^{N-1} F\left(t, d_{1} \mid\right)\left(\frac{\alpha_{1}}{2}\right)^{-\lambda} d t\right)^{\frac{1}{p-1}} d s<\frac{\alpha_{1}}{2} .
\end{aligned}
$$


This contradiction implies $w_{\alpha_{1}}(r)>\frac{\alpha_{1}}{2}, r \in[0,1)$. Thus $\alpha_{1} \in \bar{S}$,i.e. $\bar{S}$ is not empty.

(ii). $\underline{S}$ is not empty.

Let

$$
\int_{0}^{\frac{1}{2}}\left(\int_{0}^{s}\left(\frac{t}{s}\right)^{N-1} F(t, 0) d t\right)^{\frac{1}{p-1}} d s=k .
$$

Choose arbitrarily a positive number $\alpha^{\prime}$ such that $\alpha^{\prime}<\min \{k, 1\}$, we have

$$
\int_{0}^{\frac{1}{2}}\left(\int_{0}^{s}\left(\frac{t}{s}\right)^{N-1} F(t, 0)\left(\alpha^{\prime}\right)^{-\lambda} d t\right)^{\frac{1}{p-1}} d s>k>\alpha^{\prime} .
$$

Then for each $\alpha^{\prime}, w_{\alpha^{\prime}}(r)$ must vanish before $r=\frac{1}{2}$. In fact, if $w_{\alpha^{\prime}}(r)$ can be prolonged to $r=\frac{1}{2}$ and $w_{\alpha^{\prime}}\left(\frac{1}{2}\right)>0$. Since $w_{\alpha^{\prime}}(r) \leq \alpha^{\prime}, \quad r \in\left[0, \frac{1}{2}\right]$, by $(2.3),(2.4)$ and $(2.12)$, we obtain

$$
\begin{aligned}
& w_{\alpha^{\prime}}\left(\frac{1}{2}\right)=\alpha^{\prime}-\int_{0}^{\frac{1}{2}}\left(\int_{0}^{s}\left(\frac{t}{s}\right)^{N-1} F\left(t,\left|w_{\alpha^{\prime}}^{\prime}(t)\right|\right) w_{\alpha^{\prime}}^{-\lambda}(t) d t\right)^{\frac{1}{p-1}} d s \\
& \leq \alpha^{\prime}-\int_{0}^{\frac{1}{2}}\left(\int_{0}^{s}\left(\frac{t}{s}\right)^{N-1} F(t, 0)\left(\alpha^{\prime}\right)^{-\lambda} d t\right)^{\frac{1}{p-1}} d s \\
& <\alpha^{\prime}-\alpha^{\prime}=0 .
\end{aligned}
$$

This contradiction implies $\alpha^{\prime} \in \underline{S}$.

(iii). inf $\bar{S}$ does not belong to $\bar{S}$.

Put $\alpha_{*}=\inf \bar{S} \in \bar{S}$, it is clear that $\alpha_{*} \in(0, \infty)$. Suppose that $\alpha_{*} \in \bar{S}$, then $w_{\alpha_{*}}(1)=l>0$. Using condition $\left(A_{3}\right)$, for $\left(\frac{l}{2}\right)^{\frac{p+\lambda-1}{p-1}}>0$, there exists $\delta_{1}>0$ and choosing $r_{1} \in(0,1)$ sufficiently close to 1 , satisfying $1-\delta_{1}<r_{1}<1$, so that

$$
\left.\int_{r_{1}}^{1}\left(\int_{0}^{s}\left(\frac{t}{s}\right)^{N-1} F(t, d)\left(\frac{l}{2}\right)^{-\lambda}\right) d t\right)^{\frac{1}{p-1}} d s<\left(\frac{l}{2}\right)^{\frac{p+\lambda-1}{p-1}} \cdot\left(\frac{l}{2}\right)^{\frac{-\lambda}{p-1}}=\frac{l}{2}
$$

which hold uniformly for every $d \geq 0$. Since $w_{\alpha_{*}}^{\prime}(r)<0,(0<r<1)$, we get that $w_{\alpha_{*}}\left(r_{1}\right)>l$. Noting the continuous dependence of solutions of $(2.3),(2.4)$ on initial data, for all $\alpha_{0} \in\left(0, \alpha_{*}\right)$ sufficiently close to $\alpha_{*}, w_{\alpha_{0}}(r)$ are define on $\left[0, r_{1}\right]$ and satisfy

$$
w_{\alpha_{0}}\left(r_{1}\right)>l \text {. }
$$

Now we claim that such a $w_{\alpha_{0}}(r)$ satisfies $w_{\alpha_{0}}(r)>\frac{l}{2}$ on its interval of existence and, consequently, can be extended to $[0,1)$. In fact if this is not true, then, there is $r_{2} \in\left(r_{1}, 1\right)$ such that

$$
\begin{aligned}
& \frac{l}{2}=w_{\alpha_{0}}\left(r_{2}\right)=w_{\alpha_{0}}\left(r_{1}\right)-\int_{r_{1}}^{r_{2}}\left(\int_{0}^{s}\left(\frac{t}{s}\right)^{N-1} F\left(t,\left|w_{\alpha_{0}}^{\prime}(t)\right|\right) w_{\alpha_{0}}^{-\lambda}(t) d t\right)^{\frac{1}{p-1}} d s \\
& \geq l-\int_{r_{1}}^{r_{2}}\left(\int_{0}^{s}\left(\frac{t}{s}\right)^{N-1} F\left(t, d_{0}\right)\left(\frac{l}{2}\right)^{-\lambda} d t\right)^{\frac{1}{p-1}} d s \\
& >l-\int_{r_{1}}^{1}\left(\int_{0}^{s}\left(\frac{t}{s}\right)^{N-1} F\left(t, d_{0}\right)\left(\frac{l}{2}\right)^{-\lambda} d t\right)^{\frac{1}{p-1}} d s \\
& >l-\frac{l}{2}=\frac{l}{2},
\end{aligned}
$$

where $d_{0}=\max _{r_{1} \leq t \leq r_{2}}\left|w_{\alpha_{0}}^{\prime}(t)\right|$. Therefore, $\alpha_{0} \in \bar{S}$ and $\alpha_{0}<\alpha_{*}$. This contradicts the definition $\alpha_{*}=\inf \bar{S}$. Thus, inf $\overline{\bar{S}}$ does not belong to $\bar{S}$.

(iv). $\sup \underline{S}$ does not belong to $\underline{S}$.

Suppose that $\alpha^{*}=\sup \underline{S} \in \underline{S}$. Let $r_{1}$ be a point in $(0,1)$ such that $w_{\alpha^{*}}\left(r_{1}\right)=0$.Choose $T \in\left(r_{1}, 1\right)$ arbitrarily and let it be fixed. Note that

$$
\int_{r_{1}}^{T}\left(\int_{0}^{s}\left(\frac{t}{s}\right)^{N-1} F(t, 0) d t\right)^{\frac{1}{p-1}} d s>0
$$


there exists $\epsilon>0$ sufficiently small such that

$$
\int_{r_{1}}^{T}\left(\int_{0}^{s}\left(\frac{t}{s}\right)^{N-1} F(t, 0)(\epsilon)^{-\lambda} d t\right)^{\frac{1}{p-1}} d s>\epsilon .
$$

By using Lemma 2.2 and the continuous dependence of solutions on initial data, we find that $w_{\beta}(r)$ exists on $\left[0, r_{1}\right]$ and satisfies $0<w_{\beta}\left(r_{1}\right)<\epsilon$ for all $\beta>\alpha^{*}$ sufficiently close to $\alpha^{*}$. Now, we assert that such a $w_{\beta}(r)$ vanishes before $t=T$. Assume on the contrary that $w_{\beta}(r)$ exists on $[0, T]$ and remains positive. Then we obtain that $0<w_{\beta}(r)<\epsilon, r_{1} \leq r \leq T$, and integrating (2.3) twice and using $(2.15),\left(A_{1}\right)$, we obtain

$$
\begin{aligned}
& w_{\beta}(T)=w_{\beta}\left(r_{1}\right)-\int_{r_{1}}^{T}\left(\int_{0}^{s}\left(\frac{t}{s}\right)^{N-1} F\left(t,\left|w_{\beta}^{\prime}(t)\right|\right) w_{\beta}^{-\lambda}(t) d t\right)^{\frac{1}{p-1}} d s \\
& \leq \epsilon-\int_{r_{1}}^{T}\left(\int_{0}^{s}\left(\frac{t}{s}\right)^{N-1} F(t, 0) \epsilon^{-\lambda} d t\right)^{\frac{1}{p-1}} d s \\
& <\epsilon-\epsilon=0 .
\end{aligned}
$$

This contradiction shows that a $\beta$ is contained in $\underline{S}$. However, this contradicts the definition of $\alpha^{*}=\sup \underline{S}$. Thus, $\sup \underline{S}$ does not belong to $\underline{S}$.

(v). $\alpha_{0}=\inf \bar{S}=\sup \underline{S}$.

It is obvious that for all $\alpha \in \bar{S}$ and for all $\beta \in \underline{S}$, then $\beta<\alpha$. Thus, $\inf \bar{S} \geq \sup \underline{S}$. Now, we claim that $\inf \bar{S}>\sup \underline{S}$ does not hold. In fact, if this is no true, denote $\alpha=\inf \bar{S}, \beta=\sup \underline{S}$, then $\alpha>\beta$. We see clearly that $\alpha, \beta$ belong neither to $\bar{S}$ or $\underline{S}$. We find that $w_{\alpha}(r), w_{\beta}(r)$ exist in $[0,1), w_{\alpha}(1)=w_{\beta}(1)=0$ from the definition of $\bar{S}$ and $\underline{S}$. Since $\alpha>\beta$, by Lemma 2.2 we have

$$
w_{\alpha}^{\prime}(r)>w_{\beta}^{\prime}(r), \quad r \in(0,1) .
$$

Thus, $w_{\alpha}(r)-w_{\beta}(r)$ is strictly increasing in $[0,1)$ and we have

$$
w_{\alpha}(1)-w_{\beta}(1)>w_{\alpha}(0)-w_{\beta}(0)=\alpha-\beta>0 .
$$

This contradiction proves that $\alpha \geq \beta$ does not hold. Thus, $\alpha_{0}=\inf \bar{S}=\sup \underline{S}$. It follows that $w_{\alpha_{0}}(r)$ is unique positive solution of class $C^{1}[0,1) \cap C[0,1]$ of problems $(2.1)-(2.2)$. This completes the proof of Lemma 2.3.

\section{The Main Result}

We consider the singular elliptic boundary value problems (1.1)-(1.2)under the following conditions:

$\left(B_{2}\right)$. There exists functions $f^{*}, f_{*}:[0,1) \times[0, \infty) \rightarrow(0, \infty), f^{*}, f_{*} \in C_{l o c}^{\theta}([0,1) \times([0, \infty))$. Both $f^{*}(t, z), f_{*}(t, z)$ are continuously differentiable in $z$, strictly increasing in $z \geq 0$ for every fixed $t \in[0,1)$ and satisfy

$$
0<f_{*}(|t|,|z|) \leq f(x, p) \leq f^{*}(|t|,|z|), \quad(x, p) \in B \times R^{N} .
$$

From [21, 23], we get the following comparison principle which plays an important role in the proof of Theorem 3.2.

Lemma 3.1. (Weak comparison principle) Let $\Omega$ be a bounded domain in $\mathbf{R}^{N}(N \geq 2)$ with smooth boundary $\partial \Omega$ and $\theta:(0, \infty) \rightarrow(0, \infty)$ is continuous and nondecreasing. Let $u_{1}, u_{2} \in W^{1, p}(\Omega)$ satisfy

$$
\int_{\Omega}\left|\nabla u_{1}\right|^{p-2} \nabla u_{1} \nabla \psi d x+\int_{\Omega} \theta\left(u_{1}\right) \psi d x \leq \int_{\Omega}\left|\nabla u_{2}\right|^{p-2} \nabla u_{2} \nabla \psi d x+\int_{\Omega} \theta\left(u_{2}\right) \psi d x,
$$


for all non-negative $\psi \in W_{0}^{1, p}(\Omega)$ satisfy

$$
u_{1} \leq u_{2} \quad \text { on } \partial \Omega,
$$

implies that

$$
u_{1} \leq u_{2} \quad \text { in } \Omega .
$$

The main results of this paper are as follows:

Theorem 3.2. Suppose that conditions $\left(B_{1}\right)$ and $\left(B_{2}\right)$ hold, $f^{*}$ and $f_{*}$ satisfy the conditions $\left(A_{2}\right)$ and $\left(A_{3}\right)$. Then, there exists a positive solution u of class $C^{1}[0,1) \cap C[0,1]$ for singular elliptic boundary value problems (1.1)-(1.2).

Proof. We consider the following boundary value problems:

$$
\begin{gathered}
\triangle_{p} u+f^{*}(|x|,|\nabla u|) u^{-\lambda}=0, \quad x \in B, \\
u=0, \quad x \in \partial B, \\
\triangle_{p} u+f_{*}(|x|, 0) u^{-\lambda}=0, \quad x \in B, \\
u=0, \quad x \in \partial B .
\end{gathered}
$$

Applying Lemma 2.3 to these problems, we see that problems (3.2)-(3.3) and (3.4)-(3.5), respectively, have positive radial solutions $\bar{u}(|x|)$ and $\underline{u}(|x|)$ of class $C_{l o c}^{2}(B) \cap C(\bar{B})$. Note that $f^{*}, f_{*} \in C_{l o c}^{2}$. The regular theorem implies that $\bar{u}, \underline{u} \in C_{l o c}^{2+\theta}(B) \cap C(\bar{B})$. It is obvious that $\bar{u}, \underline{u}$ are a supersolution and a subsolution respectively of the boundary value problems (1.1)-(1.2). We next prove that $\bar{u}(|x|) \geq \underline{u}(|x|), x \in B$. Since $\bar{u}-\underline{u}$ satisfies

$$
\begin{gathered}
\triangle_{p} \bar{u}-\triangle_{p} \underline{u}+f^{*}(|x|,|\nabla \bar{u}|)(\bar{u})^{-\lambda}-f_{*}(|x|, 0)(\underline{u})^{-\lambda}=0, \quad x \in B, \\
\bar{u}-\underline{u}=0, \quad x \in \partial B,
\end{gathered}
$$

We can change (3.6) as follows:

$$
\begin{aligned}
& \triangle_{p} \bar{u}-\triangle_{p} \underline{u}+f^{*}(|x|,|\nabla \bar{u}|)(\bar{u})^{-\lambda}- \\
& f_{*}(|x|, 0)(\underline{u})^{-\lambda}+f_{*}(|x|, 0)(\bar{u})^{-\lambda}-f_{*}(|x|, 0)(\bar{u})^{-\lambda}=0, \quad x \in B .
\end{aligned}
$$

Condition (B2)and (3.8) can be applied to obtain

$$
\triangle_{p} \bar{u}-\triangle_{p} \underline{u}+f_{*}(|x|, 0)(\bar{u})^{-\lambda}-f_{*}(|x|, 0)(\underline{u})^{-\lambda} \leq 0, \quad x \in B,
$$

Thus, we obtain

$$
\begin{gathered}
\triangle_{p} \bar{u}-\triangle_{p} \underline{u}+c(x)(\bar{u}-\underline{u}) \leq 0, \quad x \in B, \\
c(x)=-\lambda f_{*}(|x|, 0) \int_{0}^{1}(t \bar{u}+(1-t) \underline{u})^{-(\lambda+1)} d t \leq 0 .
\end{gathered}
$$

Making use of (3.10), (3.7) and Lemma 3.1, we have

$$
\bar{u}(|x|) \geq \underline{u}(|x|), \quad x \in B .
$$

Let $B_{n}=\left\{x \in R^{N}|| x \mid<1-\frac{1}{n}\right\}$ for $n=2,3, \ldots$, and $h$ be a function of class $C_{\text {loc }}^{2+\theta}(B) \cap C(\bar{B})$ satisfying $\underline{u}(|x|) \leq h(x) \leq \bar{u}(|x|)$ in B. Since $\bar{u}(|x|)$ and $\underline{u}(|x|)$ are a supersolution and a subsolution respectively, of boundary value problems (1.1)-(1.2), we see clearly that $\bar{u}, \underline{u}$ are also a supersolution and a subsolution of the following boundary value problems

$$
\left.\triangle_{p} u+f(x, \nabla u)\right) u^{-\lambda}=0, \quad x \in B_{n},
$$




$$
u=h(x), \quad x \in \partial B_{n}
$$

for each $n \geq 2$, and satisfy

$$
\bar{u}(|x|) \geq \underline{u}(|x|), \quad x \in \bar{B}_{n} .
$$

Using condition $\left(B_{1}\right)$ and Lemma 2.1 , we find that there exists a positive $u_{n} \in C_{l o c}^{1+\theta}\left(\bar{B}_{n}\right)(n \geq 2)$ for boundary value problems (3.13)-(3.14) and satisfy

$$
\bar{u}(|x|) \geq u_{n}(x) \geq \underline{u}(|x|), \quad x \in \bar{B}_{n} .
$$

Now, we want to apply elliptic interior estimates together with a diagonal process to conclude: $\left\{u_{k}: k \geq 1\right\}$ has a subsequence $\left\{u_{k_{i}}: k_{i} \uparrow \infty\right\}$ such that $\left\{u_{k_{i}}\right\}$ converges to a function $u$ in $\mathrm{B}$ (pointwise) and this convergence is in $C^{1}$ on every compact set in B. (Therefore, $u \in C^{1}$ and $\left.\triangle_{p} u+f(x, \nabla u)\right) u^{-\lambda}=0$ in $B$ with $u=0$ on $\partial B$, and this concludes the proof.)

Step 1. On $B_{2},\left\{u_{k}: k \geq 2\right\}$ is uniformly bounded by $\underline{u}(x)$ and $\bar{u}(x)$. Since both $\underline{u}(x)$ and $\bar{u}(x)$ are bounded functions on $B_{2}$, there exists $M>0$ such that

$$
\left\|u_{k}(x)\right\|_{L^{\infty}\left(B_{2}\right)} \leq M
$$

for all $k \geq 2$.

From (1.1), $u_{k}$ satisfies

$$
\int_{B_{2}}\left|\nabla u_{k}\right|^{p} \leq-\int_{B_{2}} f\left(x, \nabla u_{k}\right)\left(u_{k}\right)^{-\lambda} u_{k}
$$

Therefore,

$$
\int_{B_{2}}\left|\nabla u_{k}\right|^{p} \leq M\left(\operatorname{meas} B_{2}\right)^{1 / q^{\prime}} C_{1}\left\|\nabla u_{k}\right\|_{p} .
$$

Here $1 / q^{\prime}+1 / p=1$, and $C_{1}$ is the Sobolev embedding constant. So, $\left\|u_{k}\right\|_{1, p} \leq C_{2}$. When $1<m<N$, the embedding of $W_{0}^{1, p}\left(B_{2}\right)$ in $L^{N p /(N-p)}\left(B_{2}\right)$ implies that $u_{k} \in L^{N p /(N-p)}\left(B_{2}\right)$. Applying Theorem 7.1 in [26], Page 286-287, we obtain the estimate

$$
\sup \left\{\left|u_{k}\right| ; x \in B_{2}\right\} \leq C_{3},
$$

here $C_{3}=C_{3}\left(\|\psi\|_{0}\right)$. If $p \geq N$, we get (3.15) from the Sobolev embedding theorem. Using Theorem 1.1 in[26], Page 251, we see that $u_{k}$ belongs to $C^{\alpha}\left(\bar{B}_{2}\right)$ for some $0<\alpha<1$, and

$$
\left\|u_{k}\right\|_{C^{\alpha}} \leq C_{4}
$$

here $C_{4}$ is determined by $C_{3}$. By Proposition 3.7 in [27], Page 806, we also know that $u_{k}$ belongs to $C^{2, \alpha}\left(\overline{B_{2}}\right)$ and

$$
\left\|u_{k}\right\|_{C^{1, \alpha}} \leq C_{5}
$$

Here $C_{5}$ is determined by $C_{4}$.

From the arguments above we see that there exists $C>0$ such that

$$
\left\|u_{k}\right\|_{C^{1+\alpha}\left(B_{1}\right)} \leq C, \text { for all } k \geq 2 .
$$

Since the embedding $C^{1+\alpha}\left(B_{1}\right) \rightarrow C^{1}\left(B_{1}\right)$ is compact, there exists a sequence denoted by $\left\{u_{k_{1 j}}\right\}_{j=1,2 \ldots}$ (where $k_{1 j} \uparrow \infty$ ), which converges in $C^{1}\left(B_{1}\right)$. Let $u_{1}(x)=\lim _{j \rightarrow \infty} u_{k_{1 j}}(x)$, for $x \in B_{1}$; then $u_{1}$ is a solution of $(1.1)$ with $\underline{u}(x) \leq u_{1} \leq \bar{u}(x)$. 
Step 2. Repeat Step 1 up to the existence of the sequence $\left\{u_{k_{1 j}}\right\}_{j=1,2 \ldots}$ to get a subsequence $\left\{u_{k_{2 i}}\right\}_{i=1,2 \ldots}$ converging in $C^{1}\left(B_{2}\right)$ to a limit $u_{2}$. Then likewise $u_{2}$ is a solution of $(2.4),(2.5)$ and $\left.u_{2}\right|_{B_{1}}=u_{1}$. Repeat Step 1 again on $B_{3}, \ldots$, etc. In this way, we obtain a sequence $\left\{u_{k_{n j}}\right\}_{j=1,2 \ldots}$ which converges in $C^{1}\left(B_{k}\right)$ and is a subsequence of $\left\{u_{k_{(n-1) j}}\right\}_{j=1,2 \ldots}$. Let $u_{k}=\lim _{j \rightarrow \infty} u_{k_{n j}}$, then, $u_{k}$ is a solution of (3.13), (3.14) in $B_{k}$ and $\left.u_{k}\right|_{B_{k-1}}=u_{k-1}$.

Step 3. By a diagonal process, $\left\{u_{k_{n n}}\right\}_{n=1,2 \ldots}$ is a subsequence of $\left\{u_{k_{n j}}\right\}_{j=1,2 \ldots}$ for every $n$. Thus, on $B_{k}$ for each $k$ we have

$$
\lim _{n \rightarrow \infty} u_{k_{n n}}=u_{k}
$$

So, if we define $u(x)=\lim _{n \rightarrow \infty} u_{k_{n n}}(x)$, then $u(x)$ satisfies

$$
\left.\triangle_{p} u+f(x, \nabla u)\right) u^{-\lambda}=0,
$$

and $\underline{u} \leq u(x) \leq \bar{u}$ (since $\underline{u} \leq u_{k}(x) \leq \bar{u}$ ) for every $k$. This complete the proof of Theorem 3.1.

Now, we give an example below to show the application of Theorem 3.2. The principal part of the equation below is p-Laplacian and the nonlinear function $f(x, z)$ has singularity at the boundary of the unit N-ball and isn't increasing in $z \geq 0$.

Example 3.3. Consider the singular boundary value problem

$$
\left\{\begin{array}{l}
\left(r^{N-1}\left|u^{\prime}(r)\right|^{p-2} u^{\prime}(r)\right)^{\prime}+\frac{r^{N-1}\left(2-\frac{3}{2} e^{\left.-\left|u^{\prime}(r)\right|^{2}+e^{-2\left|u^{\prime}(r)\right|^{2}}\right)}\right.}{u(r)\left(1-r^{2}\right)^{\frac{1}{2}}}=0, \quad r \in[0,1) \\
u(r)>0, r \in[0,1) \\
u^{\prime}(0)=0, u(1)=0
\end{array}\right.
$$

where $p \geq 2$.

Here

$$
f(r, z)=\frac{2-\frac{3}{2} e^{-z^{2}}+e^{-2 z^{2}}}{\left(1-r^{2}\right)^{\frac{1}{2}}}:[0,1) \times[0, \infty) \rightarrow(0, \infty),
$$

where $z=\left|u^{\prime}(r)\right|$. We take

$$
f_{*}(r, z)=\frac{2-\frac{3}{2} e^{-z^{2}}}{\left(1-r^{2}\right)^{\frac{1}{2}}}, f^{*}(r, z)=\frac{2-\frac{1}{2} e^{-z^{2}}}{\left(1-r^{2}\right)^{\frac{1}{2}}} .
$$

It is easy to check that $f(r, z)$ isn't increasing in $z \geq 0$, condition $\left(B_{2}\right)$ is satisfied and $f^{*}, f_{*}$ satisfy the condition $\left(A_{1}\right)$. Now, we check $f^{*}, f_{*}$ also satisfy conditions $\left(A_{2}\right)$ and $\left(A_{3}\right)$.

$\left(A_{2}\right)$

$$
\begin{aligned}
& \int_{0}^{1}\left(\int_{0}^{s}\left(\frac{t}{s}\right)^{N-1} f_{*}(t, d) d t\right)^{\frac{1}{p-1}} d s \\
& =\int_{0}^{1}\left(\int_{0}^{s}\left(\frac{t}{s}\right)^{N-1} \frac{\left(2-\frac{3}{2} e^{-d^{2}}\right)}{\left(1-t^{2}\right)^{\frac{1}{2}}} d t\right)^{\frac{1}{p-1}} d s \\
& \leq \int_{0}^{1}\left(\int_{0}^{s} \frac{2}{\left(1-t^{2}\right)^{\frac{1}{2}}} d t\right)^{\frac{1}{p-1}} d s \\
& \leq\left(\int_{0}^{1} \int_{0}^{s} \frac{2}{\left(1-t^{2}\right)^{\frac{1}{2}}} d t d s\right)^{\frac{1}{1 /(p-1)}} \\
& \leq\left(\int_{0}^{1} \frac{2 s}{\left(1-s^{2}\right)^{\frac{1}{2}}} d s\right)^{\frac{1}{p-1}}=2^{1 /(p-1)} .
\end{aligned}
$$

From the above compute, we know that $f_{*}$ satisfy the condition $\left(A_{2}\right)$. By the same way,we can check that $f^{*}$ satisfy the conditions $\left(A_{2}\right)$.

$\left(A_{3}\right)$ When $0<r<1$, similar to the above computation, we have 


$$
\begin{aligned}
& \int_{r}^{1}\left(\int_{0}^{s}\left(\frac{t}{s}\right)^{N-1} f_{*}(t, d) d t\right)^{\frac{1}{p-1}} d s \\
& =\int_{r}^{1}\left(\int_{0}^{s}\left(\frac{t}{s}\right)^{N-1} \frac{\left(2-\frac{3}{2} e^{-d^{2}}\right)}{\left(1-t^{2}\right)^{\frac{1}{2}}} d t\right)^{\frac{1}{p-1}} d s \\
& \leq\left(\int_{r}^{1} \frac{2 s}{\left(1-s^{2}\right)^{\frac{1}{2}}} d s\right)^{\frac{1}{p-1}}=2^{1 / p-1}\left(1-r^{2}\right)^{\frac{1}{p-1}} \leq \epsilon,
\end{aligned}
$$

when $r \geq\left(1-\frac{\epsilon^{p-1}}{2}\right)^{1 / 2}$. Choosing $\delta=1-\left(1-\frac{\epsilon^{p-1}}{2}\right)^{1 / 2}, f_{*}$ satisfy the condition $\left(A_{3}\right)$. The same result can be attained by $f^{*}$. Therefore, by Theorem 3.2, problem (3.16) has a positive solution in the unit N-ball.

\section{Acknowledgements:}

This project was supported by the National Natural Science Foundation of China(No.11171092); and by the Natural Science Foundation of the Jiangsu Higher Education Institutions of China (No.08KJB110005).

\section{References}

[1] Ahmed Mohammed,Positive solutions of the p-Laplace equation with singular nonlinearity, J. Math. Anal. Appl.352(2009)234-245. 1

[2] G. Astrita, G. Marrucci,Principles of Non-Newtonian Fuid Mechanics, McGraw-Hill,New York, 1974. 1

[3] B.Gidas, W.M. Ni, L. Nirenberg,Symmetry and related properties via the maximum principal, Comm.Math.Phys. 68(1979)209-243. 1

[4] David Arcoya ,JosCarmona, Tommaso Leonori,PedroJ.Martnez-Aparicio, Luigi Orsina, Francesco Petitta, Existence and nonexistence of solutions for singular quadratic quasilinear equations, J. Differential Equations 246(2009) 4006-4042. 1

[5] Zongming Guo, Some existence and multiplicity results for a class of quasilinear elliptic eigenvalue problems, Nonlinear Anal. 18 (1992) 957-971. 1

[6] Zongming Guo, J.R.L. Webb, Uniqueness of positive solutions for quasilinear elliptic equations when a parameter is large, Proc. Roy. Soc. Edinburgh. 124A (1994) 189-198. 1

[7] D. Gilbarg, N.S. Trudinger, Elliptic Partial Differential Equations of Second Order, second ed., Springer Verlag, New York, 1983.

[8] J.R. Esteban, J.L. Vazquez, On the equation of turbulent ltration in one-dimensional porous media, Nonlinear Anal. 10 (1982) 1303-1325. 1

[9] S.M. Gomes, On a singular nonlinear elliptic problem,SIAM J. Math. Anal. 17 (6) (1986) 1359-1369. 1

[10] Yingye Xua, Luanying Liana, Lokenath Debnath, Existence of positive solutions of singular elliptic boundary value problems in a ball,Computers and Mathematics with Applications 61 (2011)1335-1341. 1

[11] Qing Miao, Zuodong Yang, Bounded positive entire solutions of singular p-Laplacian equations, Nonlinear Analysis 69(2008) 3749-3760. 2.1

[12] O.A. Ladyzhenskaya, N.N. Uraltseva, Linear and Quasilinear Elliptic Equations, Academic Press, New York, 1968.

[13] Jie Zhou, Zuodong Yang, Jianqing Zhao, Existence of singular positive solutions for a class quasilinear elliptic equations,Applied Mathematics and Computation 190 (2007) 423-431. 1, 3, 3

[14] Chan-Gyun Kim, Existence of positive solutions for singular boundary value problems involving the onedimensional p-Laplacian,Nonlinear Analysis 70 (2009) 4259-4267. 1

[15] Zu-Chi Chen, Yong Zhou, On a singular quasilinear elliptic boundary value problem in a ball,Nonlinear Analysis 45 (2001) 909 - 924. 1

[16] Zuodong Yang, Existence of positive entire solutions for singular and non-singular quasi-linear elliptic equation,Journal of Computational and Applied Mathematics 197 (2006) 355 -364. 2

[17] A.C. Lazer, P.J. Mckenna, On a singular nonlinear elliptic boundary value problem, Proc. Amer. Math. Soc. 111 (1991) 721-730. 1

[18] A.S. Kalashnikov, On a nonlinear equation appearing in the theory of non-stationary filtration, Trudy Sem. Petrovsk. 5 (1978) 60-68 (in Russian). 1

[19] H.B. Keller, D.S. Cohen, Some positone problems suggested by nonlinear heat generation, J. Math. Mech. 16 (1967) 1361-1376. 1

[20] Zheng-an Yao, Wenshu Zhou, Existence of positive solutions for the one-dimensional singular $p$ Laplacian,Nonlinear Analysis 68 (2008) 2309-2318. 1 
[21] Cunlian Liu and Zuodong Yang, Existence of large solutions for quasilinear elliptic problems with a gradient term, Applied Mathematics and Computation 192(2007),533-545. 1

[22] CJ.V.A. Goncalves, M.C. Rezende, C.A. Santos, Positive solutions for a mixed and singular quasilinear problem,Nonlinear Analysis 74 (2011) 132-140. 1

[23] Cunlian Liu and Zuodong Yang, A boundary blow-up for a class of quasilinear elliptic problems with gradient term, J.Appl. Math. Comput. 33(2010), 23-34. 1

[24] Zhijun Zhang, Boundary behavior of solutions to some singular elliptic boundary value problems, Nonlinear Analysis 69 (2008) 2293-2302. 1 .

[25] L.K. Martinson, K.B. Pavlov, Unsteady shear flows of a conducting fluid with a rheological power law, Magnit. Gidrodinamika 2 (1971) 50-58. 1

[26] O.A.Ladyzhenskaya,N.N.Ural'tseva, Linear and Quasilinear Elliptic Equations, Academic Press, New York, 1968. 1.

[27] P. Tolksdorf, On the Dirichlet problem for quasilinear equations in domains with conical boundary point, Comm. Partial Differential Equations, 8(7)(1983), 773-817. 1, 3, 3 